\title{
Critical Casimir Interactions Between Spherical Particles in the Presence of the Bulk Ordering Fields.
}

\author{
O. A. Vasilyev \\ Max-Planck-Institut für Intelligente Systeme, Heisenbergstraße 3, D-70569 Stuttgart, Germany and \\ IV. Institut für Theoretische Physik, Universität Stuttgart, \\ Pfaffenwaldring 57, D-70569 Stuttgart, Germany
}

\begin{abstract}
The spatial suppression of order parameter fluctuations in a critical media produces Critical Casimir forces acting on confining surfaces. This scenario is realized in a critical binary mixture near the demixing transition point that corresponds to the second order phase transition of the Ising universality class. Due to these critical interactions similar colloids, immersed in a critical binary mixture near the consolute point, exhibit attraction. The numerical method for computation of the interaction potential between two spherical particles using Monte Carlo simulations for the Ising model is proposed. This method is based on the integration of the local magnetization over the applied local magnetic field. For the stronger interaction the concentration of the component of the mixture that does not wet colloidal particles, should be larger, than the critical concentration. The strongest amplitude of the interactions is observed below the critical point.
\end{abstract}

PACS numbers: 05.50.+q, 05.70.Jk, 05.10.Ln

\section{INTRODUCTION}

In 1948 Hendrick Casimir predicted that in the vacuum between two parallel perfectly conducting plates an attractive force appears [1]. This force is caused by the suppression of the zero level quantum fluctuations of the electromagnetic field in the space between plates. This phenomena is known as the quantum Casimir effect.

In the vicinity of the second-order phase transition in the critical media long-range fluctuations of the order parameter arise. This phenomenon is observed, e.g., in the critical liquid binary mixture at the demixing point. Fisher and de Gennes predicted [2], that the confinement of these fluctuations produces effective forces acting on confining surfaces. The appearance of forces due to spatial suppression of fluctuations of an order parameter in a critical media is now known as the Critical Casimir (CC) effect [3-5].

The phenomenon of colloidal particle aggregation in the critical binary mixture was first reported in [6]. In the planar geometry the $\mathrm{CC}$ effect for critical binary mixtures measured experimentally via the influence on the thickness of the liquid wetting films [7]. In this case the confining parallel surfaces are substrate-liquid and liquidvapor interfaces. Later on, interaction forces between a colloidal particle and a flat substrate were measured directly [8-10]. Critical depletion in colloidal suspensions was studied experimentally $[11,12]$. The colloidal aggregation in microgravity conditions, caused by $\mathrm{CC}$ interaction, was described in Ref [13]. The controlled phase transition in colloidal suspension in the critical binary mixture was studied in [14]. In this article the interaction potential between colloidal particles was extracted from the pair correlation function. From the experimental point of view $\mathrm{CC}$ interactions provide the possibility of tuning an interaction between colloidal particles. By varying the temperature of the binary mixture in the vicinity of the consolute point it is possible to switch on interactions between colloids in controllable and reversible way.

The critical binary mixture consists of components A and $\mathrm{B}$ (with concentrations $c_{A}$ and $c_{B}=1-c_{A}$, respectively) with the critical concentration $c_{A}^{c}$ and the critical temperature $T_{c}$. The schematic phase diagram with the lower critical point (that corresponds to the waterlutidine mixture used in experiments [6, 8-10]) is shown in Fig. 1(a). The state of such a system is characterized by the reduced temperature $t_{A B}=\left(T-T_{c}\right) / T_{c}$ and chemical potentials $\mu_{A}, \mu_{B}$ for the two components $\mathrm{A}$ and B with corresponding values $\mu_{A}^{c}, \mu_{B}^{c}$ at criticality. It is convenient to represent chemical potentials as a combination of $H_{A B}=\mu_{A}-\mu_{A}^{c}-\left(\mu_{B}-\mu_{B}^{c}\right)$ (which plays a role of the bulk ordering field) and $\delta \mu=\mu_{A}+\mu_{B}-\left(\mu_{A}^{c}+\mu_{B}^{c}\right)$ (which describes the deviation of chemical potential for both components from the critical values). In the most general case, in the vicinity of the critical point the state of the binary liquid mixture is characterized by two scaling fields that are linear combinations of these three variables $t_{A B}, H_{A B}$, and $\delta \mu$ (see [15] for detailed description).

A critical binary mixture belongs to the universality class of the Ising model which state is characterized by the reduced temperature $t=\left(T-T_{c}\right) / T_{c}$ and the bulk magnetic field $H_{\mathrm{b}}$. We consider the potential difference that is proportional to the bulk field $H_{A B} \propto H_{\mathrm{b}}$ and equal values of the reduced temperatures $t_{A B}=t$.

In accordance with the scaling theory $[16,17]$ the CC interactions are characterized by the ratio of the linear size of the system and the bulk correlation length $\xi\left(t, H_{\mathrm{b}}\right)$ which is the function of the reduced temperature $t$ and the bulk field $H_{\mathrm{b}}$. For correct interpretation of experimental results we need information about CC interactions of colloids for the three-dimensional (3D) Ising universality class.

The CC force and its scaling function of the 3D Ising universality class for the film geometry and vari- 


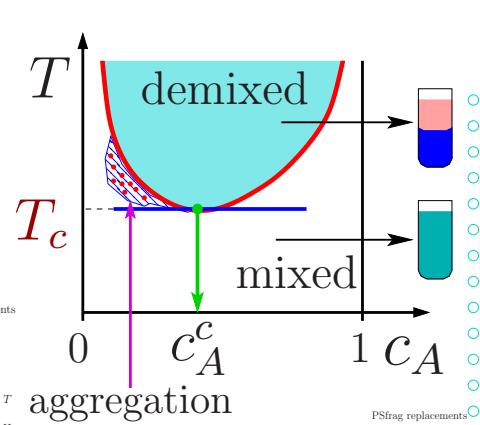

(a)

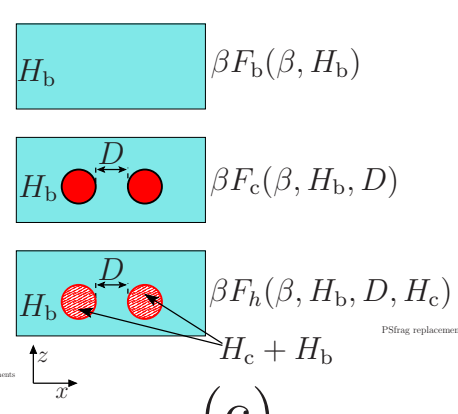

(c) $\sigma= \pm 1$ fluctuating spin

- $\sigma=+1$ frozen neighbor spin

$\begin{array}{lllllllllllll}0 & 0 & 0 & 0 & 0 & 0 & 0 & 0 & 0 & 0 & 0 & 0 & 0 \\ 0 & 0 & 0 & 0 & 0 & 0 & 0 & 0 & 0 & 0 & 0 & 0 & 0\end{array}$

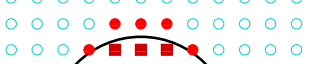

on a a a

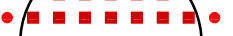

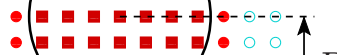

Lava a

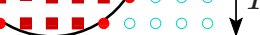

-

$\circ 0000000$

(b)

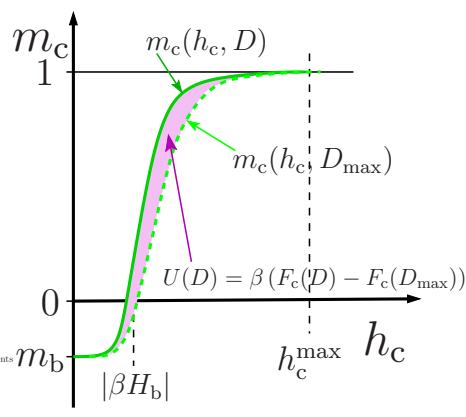

(d)
- $\sigma=+1$ frozen spin of particle

FIG. 1: (Color online) (a) Phase diagram of a critical binary mixture with the lower critical point and the aggregation region; (b) Schematic representation for a quasi-sphere on the lattice; (c) Computation of the insertion free energy: bulk system with the free energy $F_{\mathrm{b}}(\beta)$, the system with fixed spins in two colloidal particles at distance $D$ with $F_{\mathrm{c}}\left(\beta, H_{\mathrm{b}}, D\right)$, the system with an external field $H_{\mathrm{c}}$ applied to spins of two colloidal particles at distance $D$ with the free energy $F_{\mathrm{h}}\left(\beta, H_{\mathrm{b}}, D, H_{\mathrm{c}}\right)$, (d) Typical graphs of magnetizations $m_{\mathrm{c}}\left(H_{\mathrm{c}}, D\right), m_{\mathrm{c}}\left(H_{\mathrm{c}}, D_{\max }\right)$ as functions of 'colloid' field $h_{\mathrm{c}}$ for separations $D, D_{\max }$. The shadowed area between curves is equal to the absolute value of the free energy difference $U(D)=\beta F_{\mathrm{c}}(D)-\beta F_{\mathrm{c}}\left(D_{\max }\right)$.

ous boundary conditions were studied numerically without the bulk field [18-21]. Recently, Monte Carlo (MC) simulation results for the plane geometry with the bulk field were obtained $[22,23]$. Results for the CC forces between a spherical particle and a plane for the 3D Ising universality class without the bulk field are published in [24]. The CC force between two colloidal particles for the Mean Field (MF) universality class was first studied in [25] using the conformal transformation. Without the bulk field MF interactions between an elliptic particle and a wall were studied in [26] and multi-particle interactions were studied in [27]. Recently, the results for $\mathrm{CC}$ force between two colloidal particles in the presence of the bulk ordering field for the MF universality class were published [28]. Results for CC force between two disks for the two-dimensional (2D) Ising model with the bulk field were obtained via the Derjuaguin approximation [29]. The alternative method that uses the distribution function of a mobile object position for the compu- tation of the CC interaction was recently proposed [30]. In that article the $\mathrm{CC}$ interaction potential between a disk and a wall was computed for 2D geometry.

In the present paper we propose the numerical method for the direct computation of $\mathrm{CC}$ interactions between particles for a 3D Ising model with the bulk ordering field. We present results for the interaction potential for two particles as a function of the bulk field at fixed temperatures and as functions of the temperature for fixed values of $H_{\mathrm{b}}$. The paper is organized as follows: in the second section we describe the numerical method. In the third section results of the MC simulation for the interaction energy between two particles are presented. The last section is the conclusion.

\section{METHOD}

We consider the Ising model on a simple cubic lattice with periodic boundary conditions, all distances are measured in lattice units. The system size is $L_{x} \times L_{y} \times L_{z}$. In a site $i$ of the lattice the classical spin $\sigma_{i}= \pm 1$ is located. The inverse temperature is $\beta=1 /\left(k_{\mathrm{B}} T\right)$. Our aim is to study the interaction between colloidal particles immersed in the critical binary mixture. Therefore we need the lattice representation of colloidal particles. The idea proposed by Martin Hasenbusch [24] is to draw a sphere of a certain radius $R$ around a selected spin. Then all spins within the sphere are considered to belong to the colloidal particle and fixed to be +1 . In Fig. 1(b) we plot a cross section of a sphere of the radius $R=3.5$, spins inside the sphere are denoted by filled squares. We consider the case of very strong positive surface fields for colloids. This choice corresponds to the symmetry-breaking Boundary Conditions (BCs) with completely ordered surface and usually denoted as $(++)$ BC (see [31] for details). It means, that a neighbor spin $j$, that is in a contact with a particle surface will be frozen $\sigma_{j}=+1$; such spins are denoted by filled circles. Let us denote as $\{\mathrm{col}\}$ the set of all frozen spins in the system (spins in both colloidal particles and their neighbors, totally $N_{c}$ spins) and refer to this set as spins of colloidal particles. These spins are shown by filled symbols in Fig. 1(b). Fluctuating spins in the bulk are denoted by empty circles.

Let us denote as $F_{\mathrm{b}}$ the free energy of an empty bulk system (see Fig. 1(c) top) with the standard Hamiltonian for a spin configuration $\{\sigma\}$

$$
\mathcal{H}_{\mathrm{b}}(\{\sigma\})=-J \sum_{\langle i j\rangle} \sigma_{i} \sigma_{j}-H_{\mathrm{b}} \sum_{n} \sigma_{n}
$$

where $J=1$ is the interaction constant, $H_{\mathrm{b}}$ is the bulk magnetic field, the sum $\langle i j\rangle$ is taken over all neighbor spins, the sum over $n$ is taken over all spins of the spin configuration $\{\sigma\}$. The free energy of the system is expressed via the sum over all possible spin configurations $\Omega$ 
as $F_{\mathrm{b}}\left(\beta, H_{\mathrm{b}}\right)=-\frac{1}{\beta} \log \left[\sum_{\{\sigma\} \in \Omega} \mathrm{e}^{-\beta \mathcal{H}_{\mathrm{b}}(\{\sigma\})}\right]$. The system with two colloidal particles of a radius $R$ at a distance $D$ (see Fig. 1(c) middle) is described by the same Hamiltonian Eq.(1). But all spins $\sigma_{k} \in\{\mathrm{col}\}$ of colloidal particles and their neighbors $\{\mathrm{col}\}$ should be frozen $\sigma_{k}=+1$, $k \in\{\mathrm{col}\}$, so the free energy is

$$
F_{\mathrm{c}}\left(\beta, H_{\mathrm{b}}, D\right)=-\frac{1}{\beta} \log \left[\sum_{\{\sigma\} \in \Omega} \prod_{k \in\{\mathrm{col}\}} \delta_{\sigma_{k}, 1} \mathrm{e}^{-\beta \mathcal{H}_{\mathrm{b}}(\{\sigma\})}\right] .
$$

Here the product of the Dirac delta functions $\delta_{\sigma_{k}, 1}$ fixes the values of spins in colloidal particles $k \in\{\mathrm{col}\}$ to be +1 . In this expression for a free energy we also count the interaction between frozen spins within particles. Let us consider the system with the Hamiltonian

$$
\mathcal{H}_{\mathrm{h}}(\{\sigma\})=-J \sum_{\langle i j\rangle} \sigma_{i} \sigma_{j}-H_{\mathrm{b}} \sum_{n} \sigma_{n}-H_{\mathrm{c}} \sum_{k \in\{\mathrm{col}\}} \sigma_{k},
$$

where the additional external local magnetic field $H_{\mathrm{c}}$ is applied to spins $\sigma_{k}$ of colloidal particles $k \in\{\mathrm{col}\}$ (see Fig. 1(c) bottom). The free energy of this system is given by the formula

$$
F_{\mathrm{h}}\left(\beta, H_{\mathrm{b}}, D, H_{\mathrm{c}}\right)=-\frac{1}{\beta} \log \left[\sum_{\{\sigma\} \in \Omega} \mathrm{e}^{-\beta \mathcal{H}_{\mathrm{h}}(\{\sigma\})}\right] .
$$

For zero additional field this free energy equals the free energy of the system without particles $F_{\mathrm{h}}\left(\beta, H_{\mathrm{b}}, D, H_{\mathrm{c}}=\right.$ $0)=F_{\mathrm{b}}\left(\beta, H_{\mathrm{b}}\right)$. We consider systems with certain bulk field $H_{\mathrm{b}}$ at fixed inverse temperature $\beta$. Therefore in this section we omit arguments $\left(\beta, H_{\mathrm{b}}\right)$ of functions for the simplicity of notations. For a very strong additional field $\beta H_{\mathrm{c}} \gg 1$ it has a limit $\lim _{\beta H_{\mathrm{c}} \rightarrow \infty} F_{\mathrm{h}}\left(H_{\mathrm{c}}, D\right)=$ $F_{\mathrm{c}}(D)-H_{\mathrm{c}} N_{\mathrm{c}}$, where $N_{\mathrm{c}}$ is the total number of spins in the colloidal particle $\{\mathrm{col}\}$, because these spins became frozen by the local field $H_{\mathrm{c}}$. Let us introduce the variable $h_{\mathrm{c}}=\beta H_{\mathrm{c}}$. Then the magnetization of spins in colloids $M_{\mathrm{c}}=\sum_{k \in\{\mathrm{col}\}} \sigma_{k}$ is expressed via the derivative of the free energy with respect to $h_{\mathrm{c}}$ :

$$
M_{\mathrm{c}}\left(h_{\mathrm{c}}, D\right)=-\frac{\partial\left[\beta F_{\mathrm{h}}\left(h_{\mathrm{c}} / \beta, D\right)\right]}{\partial h_{\mathrm{c}}}
$$

Introducing the normalized (per total number $N_{c}$ of spins in particles) particle magnetization $m_{\mathrm{c}}\left(h_{\mathrm{c}}, D\right)=$ $M_{\mathrm{c}}\left(h_{\mathrm{c}}, D\right) / N_{\mathrm{c}}$, we can express the free energy via an integral over the magnetization

$$
\beta F_{\mathrm{h}}\left(H_{\mathrm{c}}, D\right)=\beta F_{\mathrm{b}}-N_{\mathrm{c}} \int_{0}^{\beta H_{\mathrm{c}}} m_{\mathrm{c}}\left(h_{\mathrm{c}}, D\right) \mathrm{d} h_{\mathrm{c}} .
$$

Selecting some big enough maximal value of the additional field $h_{\mathrm{c}}^{\max } \gg 1$ we can express the free energy of the system with colloidal particles as

$$
\beta F_{\mathrm{c}}(D)=\beta F_{\mathrm{b}}+N_{\mathrm{c}} \int_{0}^{h_{\mathrm{c}}^{\max }}\left[1-m_{\mathrm{c}}\left(h_{\mathrm{c}}, D\right)\right] \mathrm{d} h_{\mathrm{c}} .
$$

The particle magnetization at zero additional field $h_{\mathrm{c}}=0$ equals the bulk magnetization $m_{\mathrm{c}}\left(h_{\mathrm{c}}=0, D\right)=m_{\mathrm{b}}$ and it is equal to 1 at strong $h_{\mathrm{c}} \gg 1$ field $\lim _{h_{\mathrm{c}} \rightarrow \infty} m_{\mathrm{c}}\left(h_{\mathrm{c}}, D\right)=1$.

For this reason the result of the integration in Eq.(7) does not depend on the upper limit of the integration for big enough $h_{\mathrm{c}}^{\max }$ (we use the value $h_{\mathrm{c}}^{\max }=5$ ). In Fig. 1(d) we schematically plot the magnetization $m_{\mathrm{c}}\left(h_{\mathrm{c}}, D\right)$ for the case of the negative bulk magnetic field $H_{\mathrm{b}}<0$. Graphically, the "insertion" free energy $\beta F_{\mathrm{c}}(D)-\beta F_{\mathrm{b}}$ equals the area between lines $m_{\mathrm{c}}(h, D)$ and 1 .

Our final aim is to compute the potential $U(D)$ of the Casimir force $f_{\mathrm{C}}(D)$ between two quasi-spherical particles at the distance $D$ expressed in units $k_{\mathrm{B}} T$. Up to a certain constant $C_{1}$ this potential may be expressed via the free energy $U(D)=\beta F_{\mathrm{c}}(D)+C_{1}$. We select this constant equal to the value (with the sign "-") of the free energy at some maximal separation $D_{\max }: C_{1}=-\beta F_{\mathrm{c}}\left(D_{\max }\right)$. Therefore $U(D)=$ $N_{\mathrm{c}} \int_{0}^{h_{\mathrm{c}}^{\max }}\left[m_{\mathrm{c}}\left(h_{\mathrm{c}}, D_{\max }\right)-m_{\mathrm{c}}\left(h_{\mathrm{c}}, D\right)\right] \mathrm{d} h_{\mathrm{c}}$. Graphically, in Fig. 1(d), this function equals the area between lines $m_{\mathrm{c}}\left(h_{\mathrm{c}}, D\right)$ and $m_{\mathrm{c}}\left(h_{\mathrm{c}}, D_{\max }\right)$ with the minus sign. This method is optimized for the computation of the potential of the Casimir interaction $U$. For the computation of the Casimir force $f_{\mathrm{C}}=-\frac{\partial U(D)}{\partial D}$ between two particles it would be preferable to use the modification of the proposed method in which we interpolate between two configurations for distances $D$ and $D-1$ by varying the local field $H_{\mathrm{c}}$.

\section{RESULTS}

We perform numerical simulations for the system of the size $78 \times 49 \times 49$. Two quasi-spherical particles of the radius $R=3.5$ are located at separation $D$ along the $x$ direction (see the $x-z$ cross section in Fig. 1(c)). For separation $D=0$ the particles are in the contact. The separation $D_{\max }=30$ is the maximal possible interparticle separation in $x$ direction for this system. For accurate integration over the particle magnetization we use the histogram reweighting technique $[32,33]$. The probability distribution $P\left(m_{\mathrm{c}}, h_{\mathrm{c}}\right)$ of the particle magnetization $m_{\mathrm{c}}$ is proportional to the exponent $P\left(m_{\mathrm{c}}, h_{\mathrm{c}}\right) \propto \exp \left(h_{\mathrm{c}} N_{\mathrm{c}} m_{\mathrm{c}}\right)$. We compute this probability distribution for 16 values of the additional field $h_{\mathrm{c}}^{j}=\{0,0.01,0.02,0.03,0.04$, $0.05,0.07,0.1,0.16,0.23,0.4,0.5,0.7,1,1.5,2.5\}$. The probability distribution for the value of the field $h_{\mathrm{c}}$ may be expressed as

$$
P\left(m_{\mathrm{c}}, h_{\mathrm{c}}\right)=\frac{1}{A} \exp \left[\left(h_{\mathrm{c}}-h_{\mathrm{c}}^{j}\right) N_{\mathrm{c}} m_{\mathrm{c}}\right],
$$



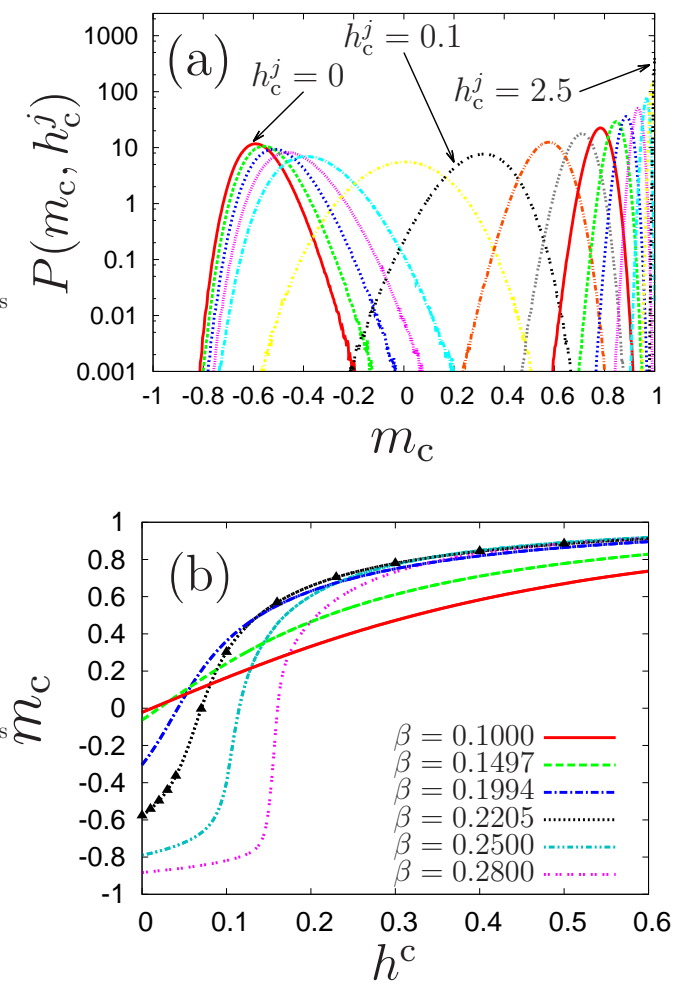

FIG. 2: (Color online) Numerical results for for the separation $D=3$ between two spheres of radius $R=3.5$, the value of the bulk field $H_{\mathrm{b}}=-0.1$. (a) Probability distribution function $P\left(m_{\mathrm{c}}, h_{\mathrm{c}}^{j}\right)$ of the magnetization $m_{\mathrm{c}}$ for the inverse temperature $\beta=0.2205$ and various values of the local field (from left to right) $h_{\mathrm{c}}^{j}=0,0.01, \ldots, 2.5$. (b) Average particle magnetization $m_{\mathrm{c}}$ as a function of the local field $h_{\mathrm{c}}$ for various values of the inverse temperature $\beta=0.1,0.1497,0.1994,0.2205,0.25,0.28$; black triangles correspond to lines from panel (a).

where the normalization constant $A=\sum_{m_{\mathrm{c}}} \exp \left[\left(h_{\mathrm{c}}-\right.\right.$ $\left.h_{\mathrm{c}}^{j}\right) N_{\mathrm{c}} m_{\mathrm{c}}$ ] and the values of fields should be close enough to let the probability distributions intersect. In Fig. 2(a) we plot the probability $P\left(m_{\mathrm{c}}, h_{\mathrm{c}}^{j}\right)$ as a function of $m_{\mathrm{c}}$ for the set of reference points $h_{\mathrm{c}}^{j}$ for $H_{\mathrm{b}}=-0.1, \beta=$ 0.2205. In Fig. 2(b) we plot the magnetization $m_{\mathrm{c}}$ as a function of $h_{\mathrm{c}}$ for $H_{\mathrm{b}}=-0.1$ and various values of $\beta=0.1,0.1497,0.1994,0.2205,0.25,0.28$. For the curve for $\beta=0.2205$ we denote by triangles values $h_{\mathrm{c}}^{j}$, for which the distribution in Fig. 2(a) is computed.

In accordance with the scaling concept the $\mathrm{CC}$ interactions between two similar colloidal particles of radius $R$ at distance $D$ at temperature $T$, and the value of the bulk field $H_{\mathrm{b}}$ are characterized by three variables: $R$, $D$ and the bulk correlation length $\xi=\xi\left(t, H_{\mathrm{b}}\right)$. Here $t=\left(T-T_{c}\right) / T_{c}=\left(\beta_{c}-\beta\right) / \beta$ is the reduced temperature $\left(\beta=1 /\left(k_{B} T\right)\right.$ is the inverse temperature). For the 3D Ising model the value of the critical inverse temperature is $\beta_{c}=0.2216544(3)$ [34]. In the general case the correlation length is an unknown function of the reduced temperature $t$ and of the bulk field $H_{\mathrm{b}}$. But for zero magnetic field the correlation length is $\xi_{t}(t) \equiv$ $\xi(t, 0)=\xi_{0}^{ \pm} t^{-\nu}$ and at the critical temperature the correlation length is $\xi_{h}\left(H_{\mathrm{b}}\right) \equiv \xi\left(0, H_{\mathrm{b}}\right)=\xi_{0}^{H}\left|H_{\mathrm{b}}\right|^{-\frac{\nu}{\Delta}}$ where the value of the correlation length critical exponent is $\nu=0.63002(10)[35], \Delta=1.5637(14)[36]$ and critical amplitudes are $\xi_{0}^{H}=0.3048(3)$ [37], $\xi_{0}^{-}=0.243(1)$, and $\xi_{0}^{+}=0.501(2)$ [34]. In the present paper we study two cases: the constant magnetic field and various temperatures and constant temperatures and various values of the magnetic field. In the first case we choose the scaling variable $r=t\left(R / \xi_{0}^{+}\right)^{1 / \nu}$ as an argument of the function because in the case of the variable $t\left(D / \xi_{0}^{+}\right)^{1 / \nu}$ for different values of $D$ we should perform computations for different temperatures (an alternative choice is the ratio $\operatorname{sgn}(t) R / \xi_{t}$, for this scaling variable the function is "stretched" in the vicinity of zero). The second reason for this choice is that it let us include the distance $D=0$ (when particles touch each other) into consideration. In the presence of the bulk ordering field, critical fluctuations on the system size scale should be suppressed, therefore in the present paper we do not study the influence of the system size.

In Figs. 3(a)-3(c) we plot the interaction potential $U\left(r ; H_{\mathrm{b}}, D\right)$ as a function of the scaling variable $r$ for separations $D=0,1,2,3,4,6$ and values of the bulk field $H_{\mathrm{b}}=0,-0.05,-0.1$, respectively. In the case of zero bulk field Fig. 3(a), the attractive potential has a pronounced minimum in the vicinity of the critical point $r \simeq 0$. For the negative value of the bulk field $H_{\mathrm{b}}=-0.05$ the amplitude of the attractive interaction increases several times. For big enough separations $D=4,6>R$ the width of the interaction potential well with respect to $r$ becomes very big. For shorter separations $D=1,2,3<R$ the minimum of the interaction disappears and the interaction within the investigated range $-4<r<14$ has no minimum. The strongest interaction corresponds to the smallest value of $r$. In Fig. 3(d) we plot the energy difference $\Delta E\left(r ; D, H_{\mathrm{b}}\right)=E\left(r ; D, H_{\mathrm{b}}\right)-E\left(r ; D_{\max }, H_{\mathrm{b}}\right)$ as a function of $r$ for separations $D=1,2,3,4,6$ with respect to maximal separation $D_{\max }=30$ (the same maximal separation is used for the computation of the interaction potential $\left.U\left(r ; D, H_{\mathrm{b}}\right)=\beta F_{\mathrm{c}}\left(r ; D, H_{\mathrm{b}}\right)-F_{\mathrm{c}}\left(r ; D_{\max }, H_{\mathrm{b}}\right)\right)$. In Figs. 4(a)-4(c) we plot the CC interaction potential $U(h ; \beta, D)$ as a function of the scaling variable $h=$ $H_{\mathrm{b}}\left(R / \xi_{0}^{H}\right)^{\Delta / \nu}$ for various separations and temperatures $\beta=0.2, \beta_{c}, 0.24$ (above $T_{c}$, at $T_{c}$, and below $T_{c}$, respectively). In Fig. 4(d) we plot the magnetization profile $m(x, z)$ as a function of coordinates $(x, z)$ for the value of the inverse temperature $\beta=0.25$ (the corresponding value of the scaling variable $r \simeq-2.48$ ) and the value of the magnetic field $H_{\mathrm{b}}=-0.1$ (the value of the scaling variable $h \simeq-42.6$ ) using the colormap. We observe, that for $D>0$ the interaction potential has a minimum as a function of $h$. The depth of this minimum decreases with increasing separation $D$. Above $T_{c}$ the minimum is smooth and is shifted for stronger negative values of 

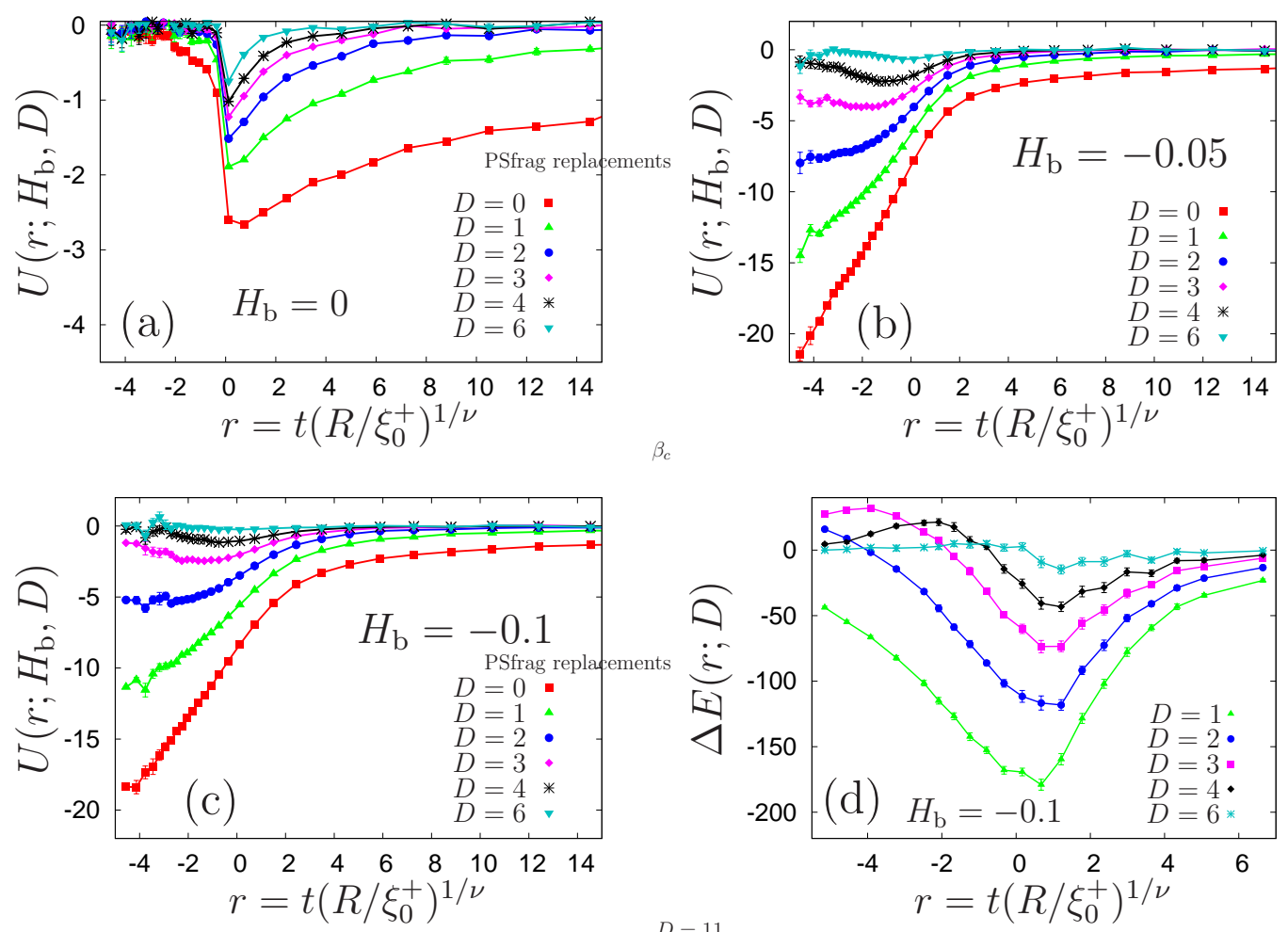

FIG. 3: (Color online) The Casimir interaction potential $U\left(r ; H_{\mathrm{b}}, D\right)$ as a function of the variable $r=t\left(R / \xi_{0}^{+}\right)^{1 / \nu}$ for various values of the separation $D=0,1,2,3,4,6$ for: (a) zero bulk field $H_{\mathrm{b}}=0$, (b) negative bulk field $H_{\mathrm{b}}=-0.05$, and (c) negative bulk field $H_{\mathrm{b}}=-0.1$. (d) The energy difference $\Delta E=E(D)-E\left(D_{\max }\right)$ as a function of the scaling variable $r$ for $H_{\mathrm{b}}=-0.1$.

$h \sim-50,-100$. Below $T_{c}$ the minima become sharp and narrow, shifted to smaller (in the amplitude) values of the negative field $-20<h<0$. In Fig. 4(d) we observe for $H_{\mathrm{b}}=0.1, \beta=0.25(h \simeq-42.6, r \simeq-2.48)$ the formation of the bridge of positive spins (which corresponds to component $A$ of the binary mixture) for small separations $D=1,3$. For larger separation $D=6$ the bridge disappears. That correlates with the presence of an attractive potential in Fig. 4(c) for $h \simeq-40$ and $D=1,3$ and the absence of attraction for $D=6$. It means, that the strong attraction for $D=1,2,3$ in Figs. 3(b) and 3(c) for $r<-4$ and in Fig. 4(c) for $h<-100$ is produced by the formation of the bridge of positive spins. This is confirmed by the energy difference $\Delta E$ in Fig. 3(d), which has the noticeable minimum for $D=1,2,3$. It corresponds to the total decreasing of the area of the -+ interface below $T_{c}$ due to the formation of the bridge. For $D=6$ the energy difference has no pronounced minimum, in this case the bridge is absent.

\section{CONCLUSION}

A numerical method for the computation of the potential of the CC interaction between particles immersed in the critical media is proposed. This method provides results for the 3D Ising universality class in the presence of non-zero bulk ordering field. The potential energy difference for two interparticle distances $D$ and $D_{\text {min }}$ has a simple graphical representation and is proportional to the area between graphs of the local magnetization for these two separations. We compute the interaction potential as a function of the temperature scaling variable for fixed values of the bulk ordering field and vice versa, as a function of the bulk field scaling variable for fixed temperatures. The strongest interaction for particles with $(+)$ boundary conditions (for colloidal particles with the surface that has a preference to component A) is observed for negative bulk fields $H_{\mathrm{b}}<0$ (B-rich phase of the binary mixture) below the critical point $T<T_{c}$ (above the lower critical point in the phase diagram Fig. 1(a)). This aggregation region is shown in Fig. 1(a) (as observed in [6]). For a small interparticle distances we observe the formation of the bridge of + phase between particles that produces forces acting far away from criticality. As a result of the computation the interaction potential between two colloidal particles is provided that is convenient for comparison with experimental results $[8,14]$. The proposed method may be applied also to studying multi-particle interactions (which play a significant role in the critical aggregation in the vicinity of the critical point [38]) in a critical solvent. 

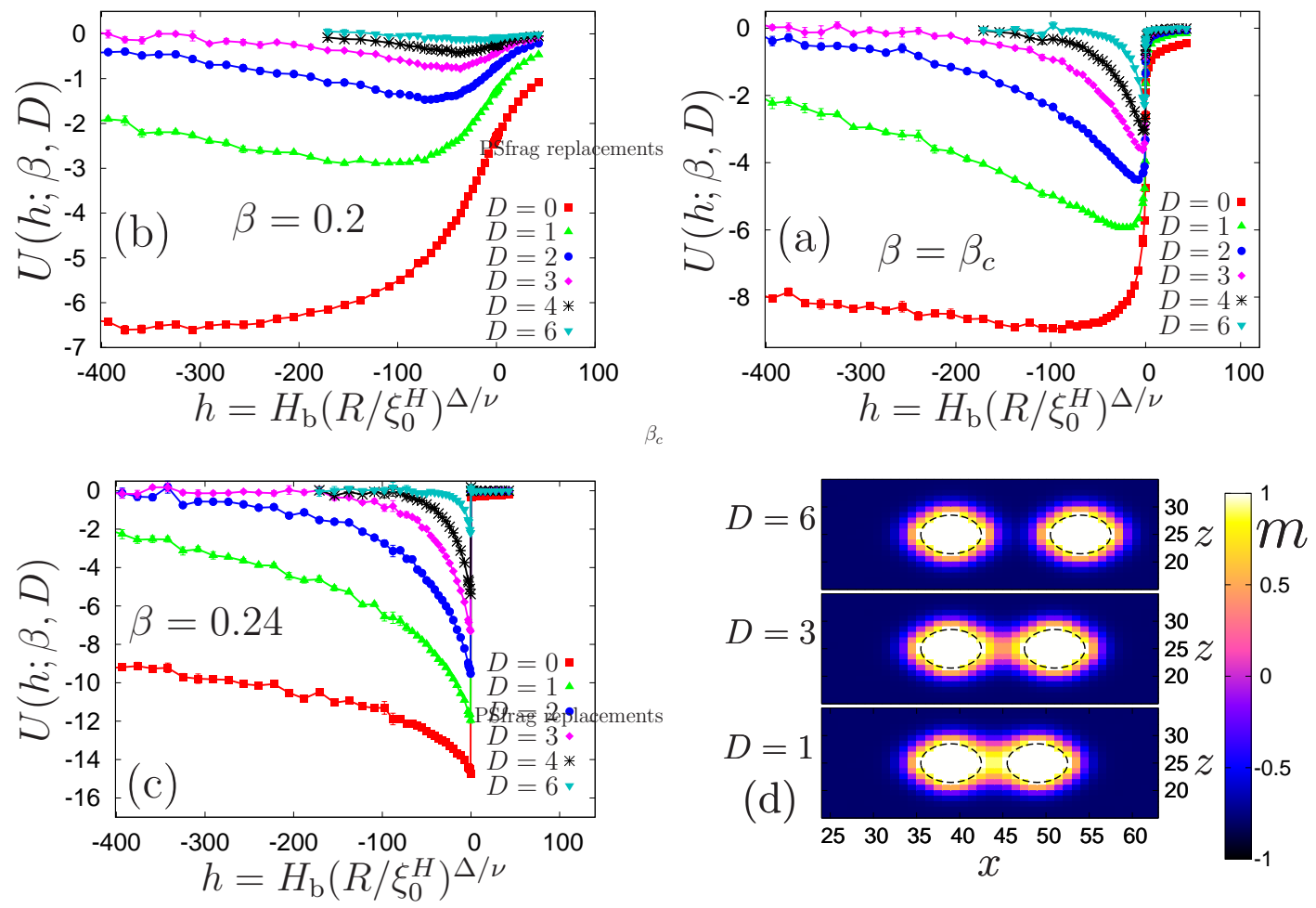

FIG. 4: (Color online) The Casimir interaction potential $U(h ; \beta, D)$ as a function of the bulk field scaling variable $h=$ $H_{\mathrm{b}}\left(R / \xi_{0}^{H}\right)^{\Delta / \nu}$ for various values of separation $D=0,1,2,3,4,6$ : (a) above the critical point $\beta=0.2$, (b) at the critical point $\beta_{c} \simeq 0.221654$, and (c) below the critical point $\beta=0.24$. (d) The magnetization profile $m(x, z)$ as a function of coordinates $x, z$ for $\beta=0.25(r \simeq-2.48), H_{\mathrm{b}}=-0.1(h \simeq-42.6)$, and various separations $D=1,3,6$. 
[1] H. B. Casimir, Proc. K. Ned. Akad. Wet. 51793 (1948).

[2] M. E. Fisher and P. G. de Gennes, C. R. Acad. Sci. Paris Ser. B 287, 207 (1978).

[3] M. Krech, Casimir Effect in Critical Systems (World Scientific, Singapore, 1994).

[4] J. G. Brankov, D. M. Dantchev, and N. S. Tonchev, The Theory of Critical Phenomena in Finite-Size Systems Scaling and Quantum Effects (World Scientific, Singapore, 2000).

[5] A. Gambassi, J. Phys.: Conf. Ser. 161, 012037 (2009).

[6] D. Beysens and D. Estève, Phys. Rev. Lett. 54, 2123 (1985).

[7] M. Fukuto, Y. F. Yano, and P. S. Pershan, Phys. Rev. Lett. 94, 135702 (2005).

[8] C. Hertlein, L. Helden, A. Gambassi, S. Dietrich, and C. Bechinger, Nature 451, 172 (2008).

[9] A. Gambassi, A. Maciołek, C. Hertlein, U. Nellen, L. Helden, C. Bechinger, and S. Dietrich, Phys. Rev. E 80, 061143 (2009).

[10] U. Nellen, L. Helden, and C. Bechinger, EPL 88, 26001 (2009).

[11] S. Buzzaccaro, J. Colombo, A. Parola, and R. Piazza, Phys. Rev. Lett. 105, 198301 (2010).

[12] R. Piazza, S. Buzzaccaro, A. Parola, and J. Colombo, J. Phys.: Condens. Matter 23, 194114 (2011).

[13] S.J. Veen, O. Antoniuk, B. Weber, M.A.C. Potenza, S. Mazzoni, P. Schall, and G.H. Wegdam, Phys. Rev. Lett. 109, 248302 (2012).

[14] V.D. Nguyen, S. Faber, Z. Hu, G.H. Wegdam, and P. Schall, Nature Comm. 4, 1584 (2013).

[15] N.B. Wilding, Phys. Rev. E 55, 6624 (1997).

[16] M. N. Barber, in Phase Transitions and Critical Phenomena, edited by C. Domb and J. L. Lebowitz (Academic, New York, 1983), Vol. 8, p. 149.

[17] V. Privman, in Finite Size Scaling and Numerical Simulation of Statistical Systems, edited by V. Privman (World Scientific, Singapore, 1990), p. 1.
[18] O. Vasilyev, A. Gambassi, A. Maciołek, and S. Dietrich, EPL 80, 60009 (2007).

[19] A. Hucht, Phys. Rev. Lett. 99, 185301 (2007).

[20] O. Vasilyev, A. Gambassi, A. Maciołek, and S. Dietrich, Phys. Rev. E 79, 041142 (2009).

[21] M. Hasenbusch, Phys. Rev. B 82, 104425 (2010).

[22] O. Vasilyev and S. Dietrich, EPL 104, 60002 (2013).

[23] D.L. Cardozo, H. Jacquin, P.C.W. Holdsworth, preprint arXiv:1404.4747 (2014).

[24] M. Hasenbusch, Phys. Rev. E 87, 022130 (2013).

[25] F. Schlesener, A. Hanke, and S. Dietrich, J. Stat. Phys. 110, 981 (2003).

[26] S. Kondrat, L. Harnau, and S. Dietrich, J. Chem. Phys. 131, 234902 (2009).

[27] T. G. Mattos, L. Harnau, and S. Dietrich, J. Chem. Phys. 138,074704 (2013).

[28] T.F. Mohry, S. Kondrat, A. Maciołek, and S. Dietrich, preprint arXiv:1403.5492 (2014).

[29] M. Zubaszewska, A. Maciołek, and A. Drzewiński, Phys. Rev. E 88, 052129 (2013).

[30] H. Hobrecht and A. Hucht, EPL 106, 56005 (2014).

[31] O. Vasilyev, A. Maciołek, S. Dietrich Phys. Rev. E 84, 041605 (2011).

[32] A. M. Ferrenberg and R. H. Swendsen, Phys. Rev. Lett. 63, 1195 (1989).

[33] D. P. Landau and K. Binder, A Guide to Monte Carlo Simulations in Statistical Physics (Cambridge University Press, London, 2005).

[34] C. Ruge, P. Zhu, and F. Wagner, Physica A 209, 431 (1994).

[35] M. Hasenbusch, Phys. Rev. B 82, 174433 (2010).

[36] A. Pelissetto and E. Vicari, Phys. Rep. 368, 549 (2002).

[37] J. Engels, L. Fromme, and M. Seniuch, Nucl. Phys. B 655, 277 (2003).

[38] M.T. Dang, A.V. Verde, V.D. Nguyen, P.G. Bolhuis, and P. Schall, J. Chem. Phys. 139, 094903 (2013). 